\title{
Leg pains, clubbing of digits and lung mass: What is your call?
}

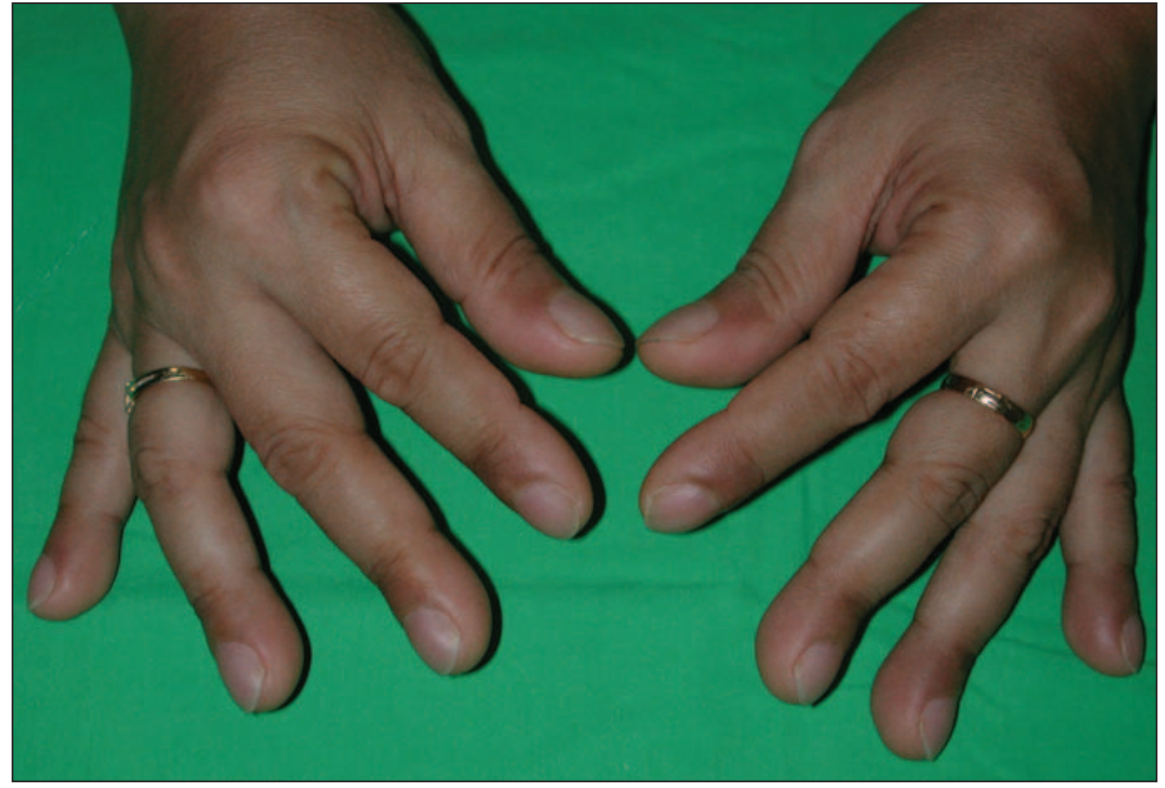

Figure 1: Clubbing of the fingers of a 43-year-old woman.

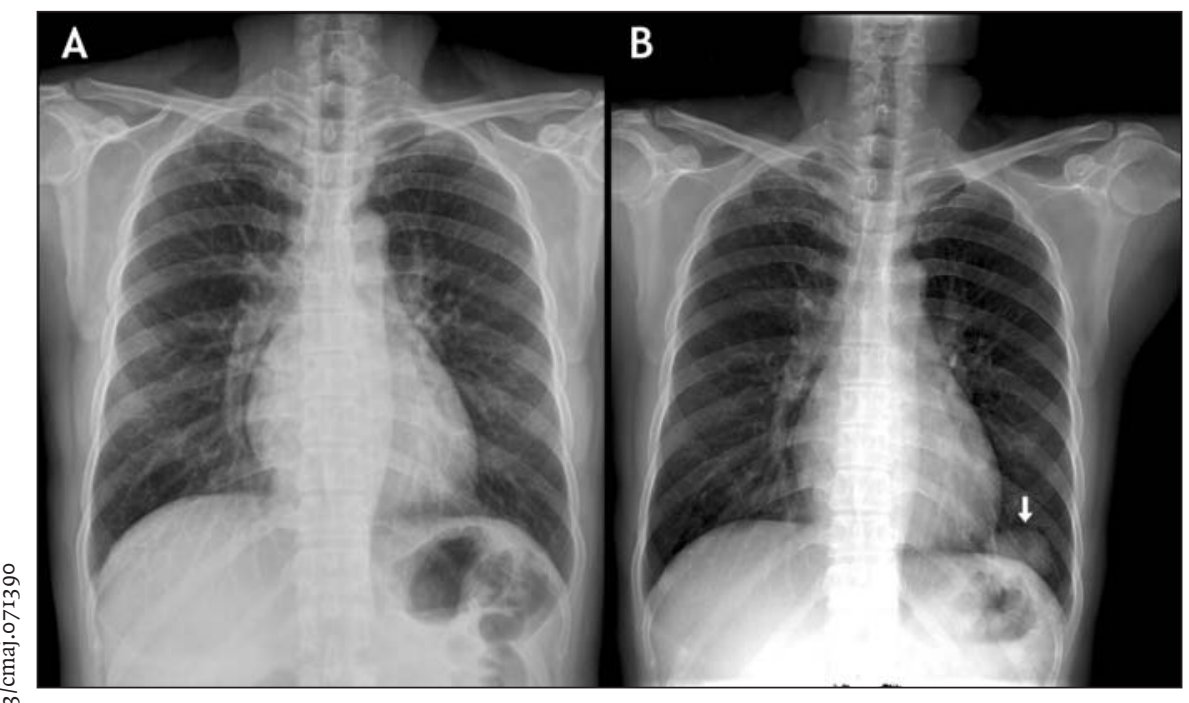

Figure 2: Chest radiographs, taken 6 months apart, with the second one (B) showing a mass in the lower lobe of the left lung (arrow).
A 43-year-old woman presented to a rheumatology clinic with a 6-month history of progressive swelling of her lower legs and pain in her knees and ankles. She had no pre-existing medical conditions and was a nonsmoker. She had not been unwell. In particular, she had no constitutional, cardiac, gastrointestinal or respiratory symptoms such as cough, fever, dyspnea or hemoptysis. On physical examination, she had bilaterally warm and tender knees and ankles, and clubbing of her fingers and toes (Figure I). The results of laboratory investigations including a complete blood count, urinalysis and hepatic and renal tests were normal. Rheumatoid arthritis was diagnosed, but therapy with oral nonsteroidal antiinflammatory drugs only marginally improved the patient's pain. The rheumatoid factor was normal, and results of tests for both antinuclear antibodies and anticyclic citrullinated peptide antibodies were negative. Because of the clubbing, we ordered pulmonary function tests and echocardiography, the results of which were normal. At follow-up 6 months later, a chest radiograph showed a large lesion in the lower lobe of the left lung (Figure 2).

\section{Which diagnosis is most likely?}

a. Granulomatous disease (e.g., Wegener granulomatosis)

b. Systemic lupus erythematosus with atypical lung involvement

c. Rheumatoid arthritis with pulmonary nodules

d. Tuberculosis (intrapulmonary and extrapulmonary)

e. Hypertrophic pulmonary osteoarthropathy 


\section{CLINICAL QUIZ}

\section{Discussion}

The answer is (e) hypertrophic pulmonary osteoarthropathy. We diagnosed pulmonary adenocarcinoma after biopsy of the lung mass. A computed tomography scan of the chest showed a pulmonary tumour $3.5 \mathrm{~cm}$ in diameter on the lower lobe of the left lung. A computed tomography scan of the patient's brain did not show a metastatic tumour, and we did not find lymph node metastases during the surgical dissection. Arthralgia of the knee and ankle joints improved 4 months after resection of the tumour.

Hypertrophic pulmonary osteoarthropathy: Hypertrophic pulmonary osteoarthropathy is a paraneoplastic syndrome characterized by clubbing of the fingers or toes, periostitis of the long bones, or arthritic symptoms in the wrists, elbows, knees and ankles. ${ }^{1}$ Radiographs of bones may disclose a solid periosteal reaction along the long bones, such as tibial, fibular or femoral shafts. Whole-body bone scintigraphy shows a characteristic pattern and is the preferred method for diagnosing the syndrome (Figure 3 ).

Hypertrophic pulmonary osteoarthropathy is often associated with primary or metastatic pulmonary cancer, most commonly primary pulmonary adenocarcinoma. ${ }^{2}$ Some abdominal cancers are also associated with the disease. Although the cause is not fully understood, increased blood flow in the long bones has been described, as well as elevations of tumour-associated factors, including growth hormone, transforming growth factor $\beta$-I, vascular endothelial growth factor and plasma endothelin. ${ }^{3}$

Treatment of hypertrophic pulmonary osteoarthropathy relies on treatment of the primary lesion. Hypertrophic pulmonary osteoarthropathy

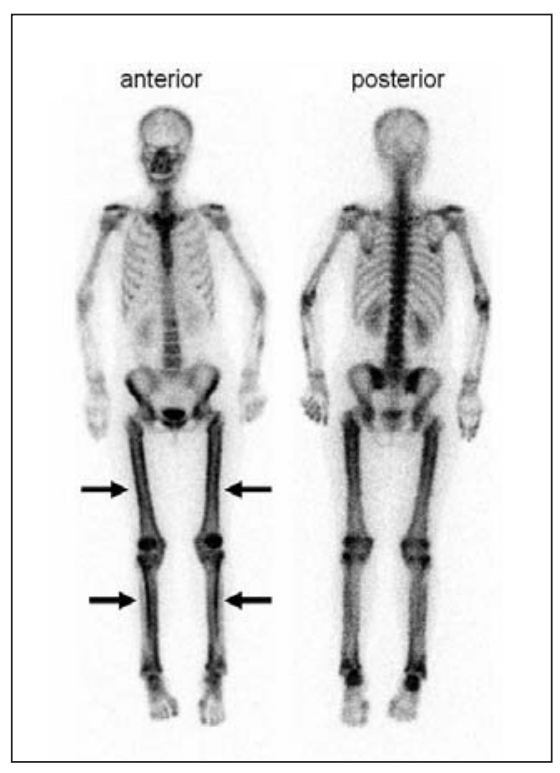

Figure 3: Whole-body bone scintigraphy showing increased tracer uptake along the cortical margins of the bilateral lower extremities (arrows), which is compatible with the "parallel stripe sign" or "tramline sign."

related to lung cancer has been reported to be improved or, in some cases, remitted completely after resection of the primary lesion.

Differential diagnosis: Granulomatous diseases, including Wegener granulomatosis, may cause local or systemic involvement and are characterized by histiocyte inflammation and granuloma formation. Wegener granulomatosis ${ }^{4}$ involves the upper and lower respiratory tracts and the kidneys. Chest radiographs may show infiltrates and nodules with or without cavities. Oral ulcers, nasal secretions, abnormal urinary sediments and serum antineutrophil cytoplasmic antibodies are diagnostic clues. Wegener granulomatosis is sometimes associated with arthralgia.

Systemic lupus erythematosus is an autoimmune disease in which polyarthritis is common. Over $90 \%$ of patients experience joint symptoms dur- ing the course of illness. If the lungs are involved, chest radiography tends to be consistent with pleural effusion, interstitial lung disease or pulmonary hemorrhage.

Rheumatoid arthritis commonly results in interstitial pulmonary abnormalities, but other lung manifestations include pleural disease, obstructions of the small or large airways, or, less frequently, pulmonary rheumatoid nodules. Anticyclic citrullinated peptide antibodies are $88 \%$ sensitive and $89 \%$ specific for rheumatoid arthritis. ${ }^{5}$

Tuberculous arthritis develops after dissemination of primary pulmonary lesions. Chest radiographs may show patterns of active or old tuberculosis such as fibrosis and calcification in the upper regions of the lungs.

\section{Jenn-Yu Wu MD \\ Jin-Yuan Shih MD PhD}

Department of Internal Medicine National Taiwan University Hospital Taipei, Taiwan

Competing interests: None declared.

\section{REFERENCES}

I. Dickinson CJ. The aetiology of clubbing and hypertrophic osteoarthropathy. Eur J Clin Invest I993;23:330-8.

2. Kishi K, Nakamura H, Sudo A, et al. Tumor debulking by radiofrequency ablation in hypertrophic pulmonary osteoarthropathy associated with pulmonary carcinoma. Lung Cancer 2002;38:317-20.

3. Martinez-Lavin M. Exploring the cause of the most ancient clinical sign of medicine: finger clubbing. Semin Arthritis Rheum 2007;36:380-5.

4. Leavitt RY, Fauci AS. Wegener's granulomatosis. Curr Opin Rheumatol I99I; 3:8-I4.

5. Suzuki K, Sawada T, Murakami A, et al. High diagnostic performance of ELISA detection of antibodies to citrullinated antigens in rheumatoid arthritis. Scand J Rheumatol 2003;32:197-204.

CMAJ invites contributions to the Clinical Quiz column, which uses multiple-choice questions to guide a focused image-based discussion of the diagnosis or management of clinical cases. Submit manuscripts online at http://mc.manuscriptcentral.com/cmaj. 